\title{
A New Approach to Estimate Hansen Solubility Parameters Using Maple Software
}

\author{
Ra'id Khider Salman ${ }^{1}$, Jassim Mohammed Salih ${ }^{2}$ \\ ${ }^{1}$ Physics Department, College of Education for Pure Sciences, University of Anbar, Anbar, Iraq \\ ${ }^{2}$ Physics Department, College Science, University of Anbar, Anbar, Iraq \\ Email address: \\ dr.raid1978@hotmail.com (R. K. Salman),dr_jassim55@yahoo.com (J. M. Salih) \\ ${ }^{*}$ Corresponding author
}

\section{To cite this article:}

Ra'id Khider Salman, Jassim Mohammed Salih. A New Approach to Estimate Hansen Solubility Parameters Using Maple Software. International Journal of Materials Science and Applications. Vol. 5, No. 5, 2016, pp. 183-187. doi: 10.11648/j.ijmsa.20160505.11

Received: July 20, 2016; Accepted: July 30, 2016; Published: August 17, 2016

\begin{abstract}
This paper represents the use of Maple software capabilities in the analyses of the polymer- solvent interactions. It illustrates the advantages of using Maple to estimate Hansen solubility parameters and to predict the polymer-solvent miscibility. The study focuses on the polymers and solvents that are employed in the polymer gel electrolytes. The acquired data showed high consistency and flexibility in calculations and estimations processes, especially in regarding with Hansen sphere plotting and relative energy distance calculations.
\end{abstract}

Keywords: Hansen Solubility Parameters, Polymer-Solvent Miscibility, Maple Software, Hansen Sphere

\section{Introduction}

Solubility parameters are a measure for the ability of the polymer to dissolve the solvent, or in general, it is the measure of the ability of any two species to be mixed. It is based originally on the cohesive energy of the material such as polymer that is affected by mixing that polymer with solvent. The classic solubility theory has been developed by Hildebrand and Scott who stated that the solubility parameter of a species $A\left(\delta_{A}\right)$ is related to its energy of vaporisation (cohesive energy) $\Delta \mathrm{E}_{\mathrm{A}}$ as follows $[1,2]$;

$$
\delta_{A}=\sqrt{\frac{\Delta E_{A}}{V_{A}}}
$$

Where $\mathrm{V}_{\mathrm{A}}$ is the molar volume of the species $\mathrm{A}$, and $\frac{\Delta E_{A}}{V_{A}}$ is known as the cohesive energy density.

$$
\Delta E=\Delta H_{v}-R T
$$

Then equation 1 can be written as:

$$
\delta=\sqrt{\frac{\Delta H_{v}-R T}{V_{A}}}
$$

Where $\Delta H_{v}, R, T$ are the heat of vaporisation, the gas constant and the absolute temperature respectively.

However, this relation seems not to be effective for polar solvents since it takes only the dispersion force effect, while neglecting the other operative forces such as polarity that plays a major role in polar solutions.

Moreover, polarity alone also is not enough to control and interpret the dissolving activity. Hydrogen bonds forces have also a great role in bonding the molecules and hence strengthen connections between the polymer chains. Therefore, solubility for swollen materials seems to be more complicated to be predicted using one reaction activity

In 1967, Hansen has developed an approach to find the solubility of solutions by using the well-known "Hansen Sphere" which is constructed by three dimensions including the three major forces that hold the molecules together and hence control the interactions between polymer and the solvent. These parameters represent the three original molecular forces that govern the dissolving activity; the dispersive force (d), the polarity (p) and the hydrogen bond energy (h) [3]. Therefore, the total cohesive energy composed of these components can be written as;

$$
\mathrm{E}=\mathrm{E}_{\mathrm{d}}+\mathrm{E}_{\mathrm{p}}+\mathrm{E}_{\mathrm{h}}
$$

Dividing equation 3 by $\mathrm{V}$ yields; 


$$
\frac{E}{V}=\frac{E_{d}}{V}+\frac{E_{p}}{V}+\frac{E_{h}}{V}
$$

Comparing equation 1 with 4 leads to [4];

$$
\delta^{2}=\delta_{d}^{2}+\delta_{P}^{2}+\delta_{h}^{2}
$$

Where $\delta_{d}, \delta_{p}$ and $\delta_{h}$ represent the three components of Hansen solubility parameters; the dispersion, the polar and the hydrogen-bonding solubility parameters.

According to these three components, any polymer can be dissolved in a solvent if their solubility parameters are close to each other. This behaviour is also related to the "like dissolves like" role. Hansen has also suggested a relation between the solubility parameters of the mixed species like polymers and solvents, which states that the distance $\mathrm{D}_{\mathrm{S}-\mathrm{P}}$ between the polymer and the solvent molecules can be given by [5];

$$
D_{S-P}=\sqrt{\left.4 *\left(\delta_{d, P}-\delta_{d, S}\right)^{2}\right)+\left(\delta_{P, P}-\delta_{P, S}\right)^{2}+\left(\delta_{h, P}-\delta_{h, S}\right)^{2}}
$$

Where $\delta_{i, P}$ and $\delta_{i, S}$ is the $\mathrm{i}$ component of solubility parameter for the polymer and the solvent respectively.

In practice, a sphere can be plotted in three dimensional space includes the three parameters $\delta_{d}, \delta_{p}$ and $\delta_{h}$ as its $\mathrm{x}, \mathrm{y}$ and $\mathrm{z}$ coordinates correspondingly. This sphere is known as Hansen sphere that has a radius $\mathrm{R}_{\mathrm{o}}$ called interaction radius which represents a unique characteristic for the required solute and can be obtained experimentally for each polymer or another solute. Hansen sphere is used to measure the miscibility of polymer in the required solvents by plotting it in $\delta_{d}, \delta_{p}$ and $\delta_{h}$ space and then plot HSPs for the other solvents as points with HSPs used as their correspond coordinates. The ratio $D_{S-\mathrm{P}} / R_{\mathrm{o}}$ is usually called as the relative energy difference RED. Therefore, the relative energy difference can be considered as one of the best approaches to estimate the miscibility of the solutions, i.e. it can give a good prediction of whether the polymer can dissolve the solvent or not according to the following criteria [3]:

If $\mathrm{D}_{\mathrm{S}-\mathrm{P}} / \mathrm{R}_{\mathrm{o}}<1$ the solvent then is good for that polymer

If $\mathrm{D}_{\mathrm{S}-\mathrm{P}} / \mathrm{R}_{\mathrm{o}}=1$ the mixture is at boundary conditions

And if $\mathrm{D}_{\mathrm{S}-\mathrm{P}} / \mathrm{R}_{\mathrm{o}}>1$ the solvent is poor for that polymer.

Under these conditions, many of researchers have investigated the compatibility of different of solvents to different polymers experimentally [6-11]. Others have used HSPs and RED to study thermodynamics of polymer solutions [10, 12-14]. On the other hand, other researchers have developed computational approaches based on the collected data out of the older experimental works to estimate the compatibility of polymers solutions and blinds $[15,16]$. Gharagheizi and Angaji have presented a computer method based on Nelder-Mead optimization algorithm to calculate HSPs for a group of polymers and solvents [17]. The results from this study have shown a good agreement with the previous experimental data. Vebber et al. have also had their contributions on calculating HSPs of polymers using computer procedure based on the genetic algorithm. Their calculations yielded a correlated data for Hansen and latter practical works.
In this study, Maple 18 software has been employed to calculate the compatibility of number of polymers with a number of solvents. Table 1 shows HSPs and $R_{0}$ for the polymers under the current study, while Table 2 shows HSPs values for the solvents. The reason behind choosing these polymers and solvents specifically is the common relation between them which is that all of these polymers and solvents are used in polymer gel electrolytes preparation [18, 19]. These types of PGEs are commonly used in lithium-ion batteries manufacturing which is the main field in the author research work.

Table 1. Hansen solubility parameters and $R_{o}$ for the polymers under study $[8,20]$.

\begin{tabular}{lllll}
\hline Polymer & $\boldsymbol{\delta}_{\mathbf{d}} \mathbf{M P a}^{\mathbf{1 / 2}}$ & $\boldsymbol{\delta}_{\mathbf{P}} \mathbf{M P a}{ }^{\mathbf{1 / 2}}$ & $\boldsymbol{\delta}_{\mathbf{h}} \mathbf{M P a}^{\mathbf{1} / 2}$ & $\mathbf{R}_{\mathbf{o}}$ \\
\hline PVDF & 17.2 & 12.1 & 10.2 & 9.6 \\
PMMA & 17.7 & 6.7 & 6.2 & 8.96 \\
PAN & 21.7 & 14.1 & 9.1 & 10.9 \\
PVC & 19.2 & 7.9 & 3.4 & 3.5 \\
\hline
\end{tabular}

Table 2. Hansen solubility parameters for the solvents that have been chosen for this study [4, 8, 9, 20].

\begin{tabular}{llll}
\hline Solvent & $\boldsymbol{\delta}_{\mathbf{d}} \mathbf{M P a}^{\mathbf{1 / 2}}$ & $\boldsymbol{\delta}_{\mathbf{p}} \mathbf{M P a}^{\mathbf{1 / 2}}$ & $\boldsymbol{\delta}_{\mathbf{h}} \mathbf{M P a}^{\mathbf{1 / 2}}$ \\
\hline PC & 20 & 18 & 4.1 \\
EC & 18 & 21.7 & 5.1 \\
DEC & 16.6 & 3.1 & 6.1 \\
DMF & 17.4 & 13.7 & 11.3 \\
GBL & 18 & 16.6 & 7.4 \\
\hline
\end{tabular}

\section{Maple Software Procedure}

The use of Maple system here to evaluate the interactions between solvents and solutes mathematically is due to its distinctive advantages. These advantages include the outstanding three dimensions graphics, the flexibility in programming and data processing, and apparently the simplicity and speed in dealing with long and complex procedures. The system is used seemingly for the first time and the procedure has been written by the author not based on previous studies; therefore, the result data have to be validated with a previous practical work. This has been already done by collecting some data from Hansen and the latter relative works [4], which will be stated accordingly in the results and discussion. Figure 1 shows flow chart of Maple procedure that has been used to estimate the miscibility of polymers in the studied solvents. The procedure starts with defining HSPs for the polymer and the solvent and the radius of Hansen sphere Ro (which has already been found either experimentally or by a reference). The processes then start by calculating the distance between the polymer and the solvent DS-P, using equation 6 . The program then starts comparing the result with Ro; if the distance is less than the radius of polymer sphere then the 
solvent is good, otherwise, there are two possibilities: one is that the distance is greater than the radius which means that the solvent is poor to dissolve that polymer and the user needs to choose another solvent (new trial by defining HSPs for another solvent). The last probability is this routine when the distance is equal to the radius, i.e. the boundary condition of the relative energy difference at which the polymer is said to be swollen rather than dissolved in the solvent. The system then uses all the nominated parameters to plot Hansen sphere for the polymer and to plot solvent parameters as straight lines which are represented by the polymer sphere centre as their start points and their HSPs as the their end points.

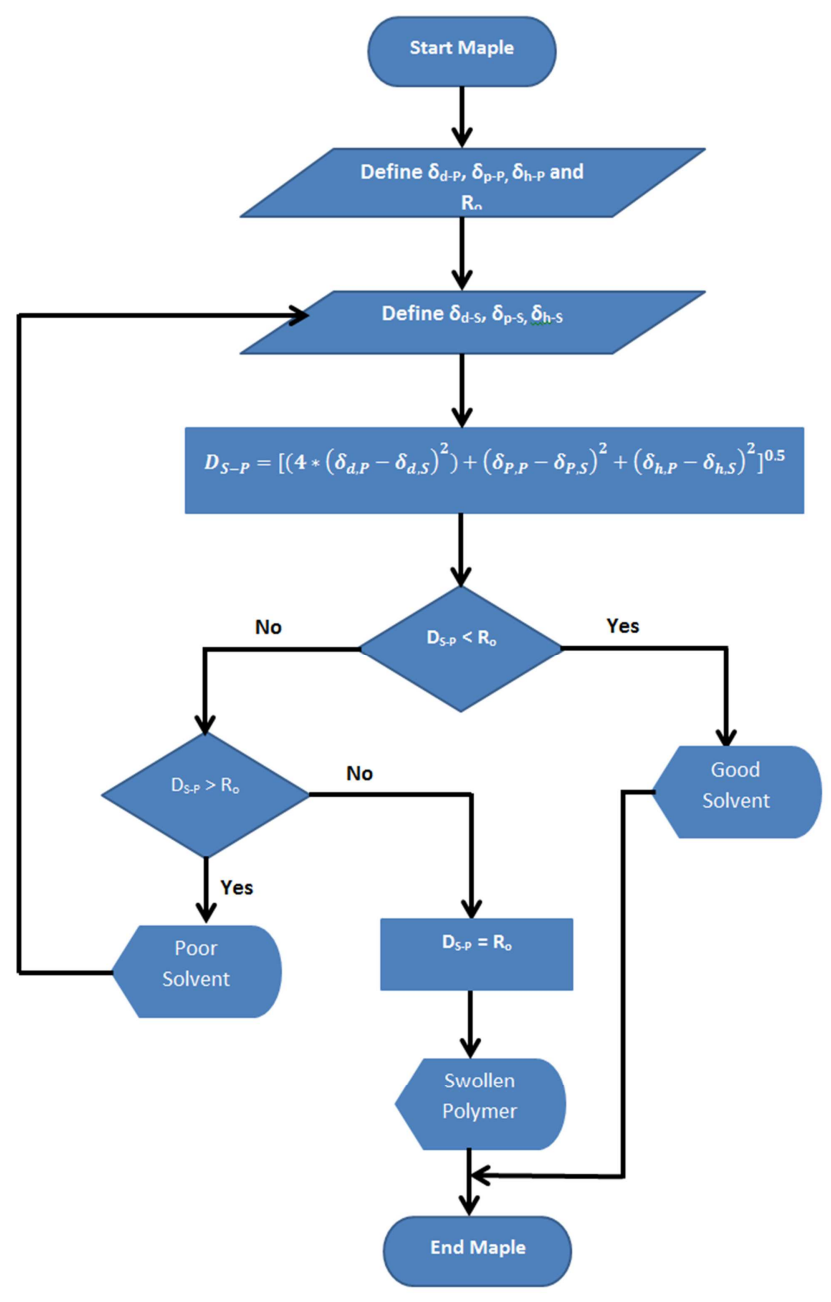

Figure 1. Flow chart of Maple procedure.

\section{Results and Discussion}

Figures from Figure 2 down to Figure 5 illustrate solubility spheres for PVDF, PMMA, PAN and PVC created by Maple procedure.

The program shows that the polymers miscibility varies in accordance with the different solubility components of the solvents. The figures show that DMF has a good compatibility for all the polymers except for PVC, which exhibits high resistance to all the tested solvents. This incompatibility is confirmed experimentally by Grause and others [21, 22].

In Figure 2, PVDF is apparently compatible with DMF and GBL. According to the technical guides and previous studies [23-26], PVDF tends to be dissolved in aprotic polar solvents very well and these two solvents have been referred as good solvents for it; meanwhile PVDF is normally resistant to ethers and esters [27-29]. This is why PVDF shows incompatibility with the other solvents. However, PC and EC are considered as latent solvents which mean that they can substantially swell PVDF at specific circumstances. This may explain why these two solvents have very short distance to PVDF sphere although they are outside it.

PMMA shows lower miscibility for DMF than DEC; meanwhile it exhibits immiscible behaviour for the other solvents. The literature states that polar dimethylformamide (DMF) dissolves PMMA in a less amount than DEC, which is evidently illustrated in Figure 3 [30-32].

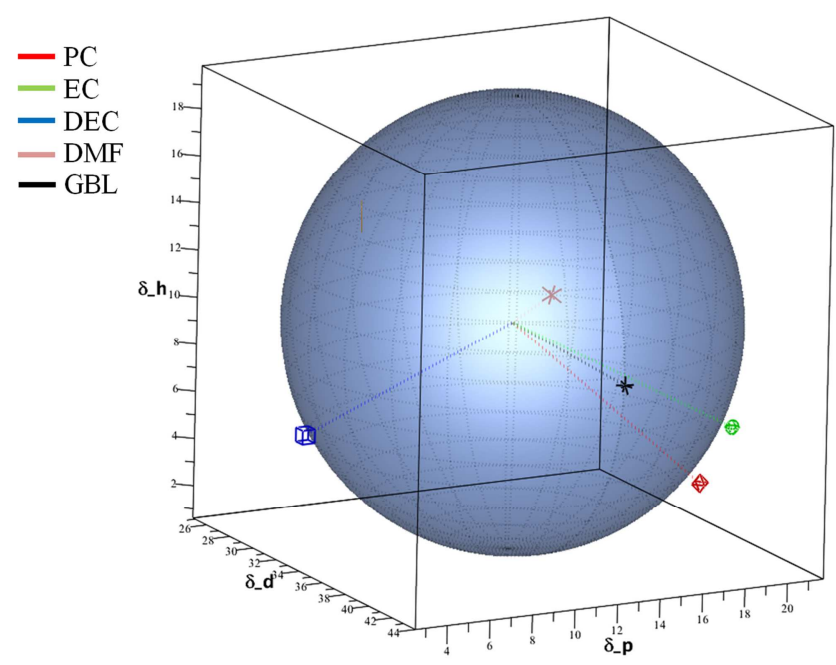

Figure 2. Solubility Sphere for PVDF.

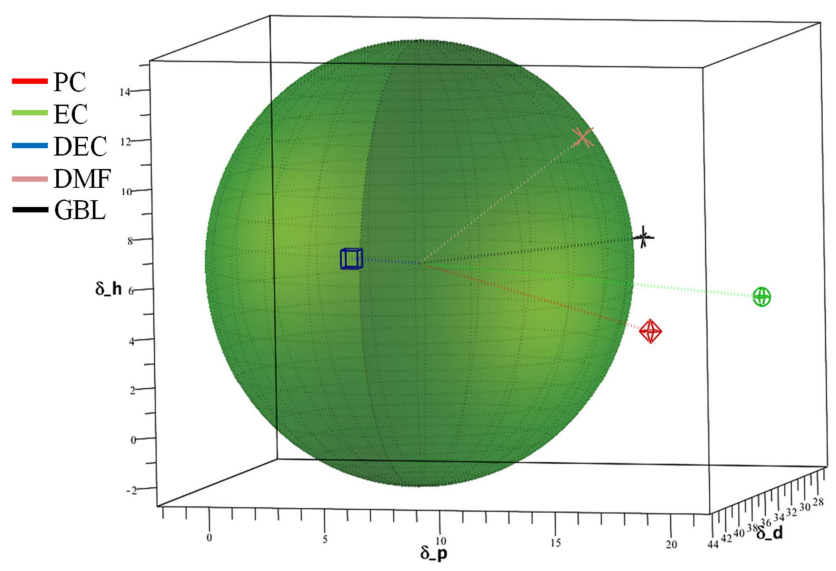

Figure 3. Solubility Sphere for PMMA.

$\mathrm{PC}$ and GBL show slightly higher dissolving power to PAN. DMF shows less power to dissolve PAN. EC and DEC show similar behaviour with PAN to that of PVDF. Similar behaviour has been reported in [33, 34].

In Figure 6 all the study species have been grouped and 
compared corresponding to the relative energy difference RED. In this figure EC has the greater RED value of the other solvents with all the polymers except for PAN, where which DEC is differed more relatively. However, its value is still by far greater than the unity. DMF has the lowest value of RED with PVDF, which again confirms the behaviour illustrated in Figure 2. The compatibility between DEC and PMMA is confirmed in this figure, likewise the large immiscibility between PMMA and PC along with EC.

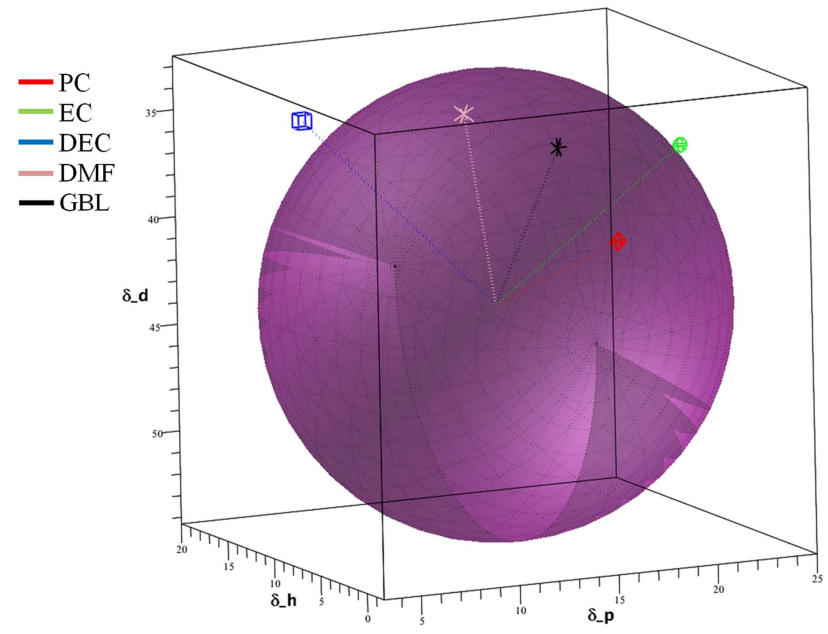

Figure 4. Solubility Sphere for PAN.

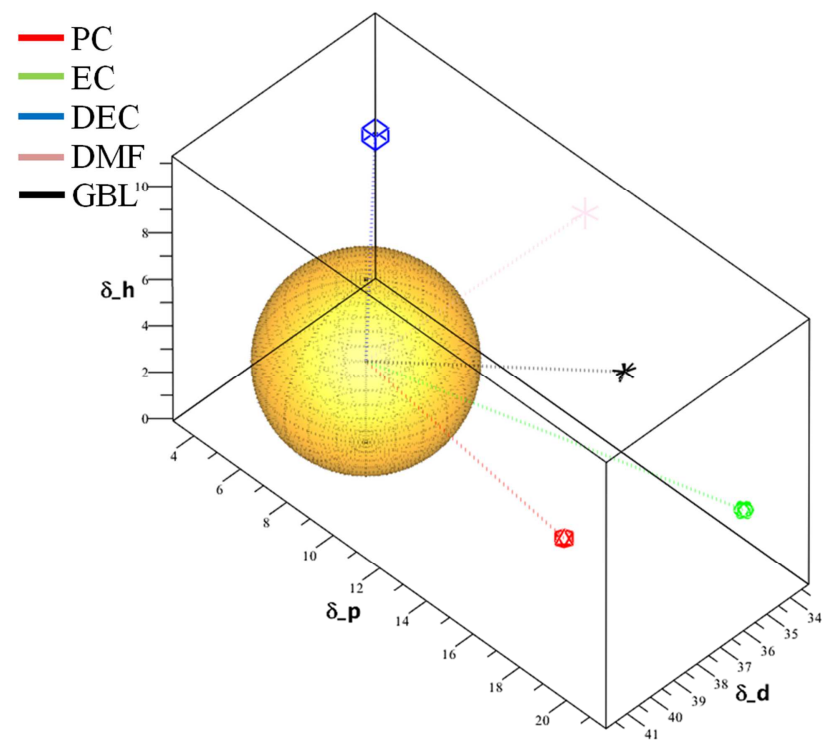

Figure 5. Solubility Sphere for PVC.

Table 3. RED values for each polymer with the corresponding solvent.

\begin{tabular}{|c|c|c|c|c|}
\hline \multirow{2}{*}{ Solvent } & \multicolumn{4}{|l|}{ RED } \\
\hline & PVDF & PMMA & PAN & PVC \\
\hline $\mathrm{PC}$ & 1.06 & 1.38 & 0.66 & 2.93 \\
\hline $\mathrm{EC}$ & 1.14 & 1.68 & 1.04 & 4.03 \\
\hline DEC & 1.04 & 0.47 & 1.4 & 2.16 \\
\hline DMF & 0.21 & 0.97 & 0.81 & 2.98 \\
\hline GBL & 0.58 & 1.11 & 0.73 & 2.82 \\
\hline
\end{tabular}

Table 4. Solvent Miscibility as Calculated By Maple.

\begin{tabular}{lll}
\hline \multirow{2}{*}{ Polymer } & \multicolumn{2}{l}{ Solvent Quality } \\
\cline { 2 - 3 } & Good & Non solvent or swelling agent \\
\hline \multirow{3}{*}{ PVDF } & DMF & PC \\
& GBL & EC \\
& & DEC \\
PMMA & DEC & PC \\
& DMF & EC \\
& PC & GBL \\
PAN & DMF & EC \\
& GBL & \\
& & PC \\
PVC & - & EC \\
& & DEC \\
& & DMF \\
\hline
\end{tabular}

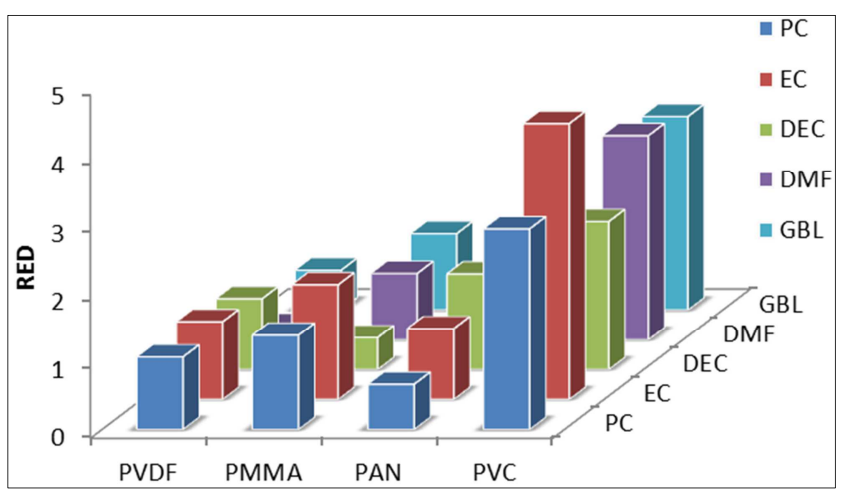

Figure 6. Comparison of calculated RED values for the study solvents in the selected polymers.

\section{Conclusion}

Maple software shows reliable calculation and data extraction in regarding with polymer-solvent interaction through Hansen solubility theory. The computed data indicate a good agreement with the validated data from earlier experimental works. Maple plots techniques shows precise and outstanding presentation for polymer sphere. Overall, Maple succeeds to present a new promises technique for this type of calculations.

\section{Acknowledgement}

The author would like to give his thanks to Professor Steven Abbott for his great help in providing HSPs for the work materials, and for his worthy advices in regarding with the HSPs calculations. The author would also like to thank Solvay (Solef) company for their help in supplying materials properties and technical information, which has had a great role on produce this paper in this professional way. 


\section{References}

[1] Hildebrand, J. H. and R. L. Scott, Solutions of Nonelectrolytes. Annual Review of Physical Chemistry, 1950. 1 (1): p. 75-92.

[2] Brown, G. H., Regular Solutions. By Joel H. Hildebrand and Robert L. Scott. Inorganic Chemistry, 1963. 2 (2): p. 431-432.

[3] Hansen, C. M., The three dimensional solubility parameter. Journal of paint technology, 1967. 39 (505): p. 104.

[4] Hansen, C. M., Hansen solubility parameters: a user's handbook. 2007: CRC press.

[5] HANSEN, C. M., THE THREE DIMENSIONAL SOLUBILITY PARAMETER AND SOLVENT DIFFUSION CO EFFICIENT, Their Importance In Surface Coating Formulation. 1967: COPENHAGEN DANISH TECHNICAL PRESS.

[6] Lee, J. N., C. Park, and G. M. Whitesides, Solvent Compatibility of Poly (dimethylsiloxane)-Based Microfluidic Devices. Analytical Chemistry, 2003. 75 (23): p. 6544-6554.

[7] Buckley-Smith, M. K., The Use of Solubility Parameters to select membrane materials for Pervaporation of organic mixtures. The University of WAIKATO, Hamilton, New Zealand, 2006.

[8] Barton, A. F. M., CRC Handbook of Solubility Parameters and Other Cohesion Parameters, Second Edition. 1991: Taylor \& Francis.

[9] Grulke, E. A., Solubility Parameter Values, in The Wiley Database of Polymer Properties. 2003, John Wiley \& Sons, Inc.

[10] Ganguly, S. C., Prediction of polymer properties. J Bicerano, Marcel Dekker, New York, 2002. ISBN 0-8247-0821-0. pp 784. Polymer International, 2005. 54 (1): p. 246-246.

[11] Alhalaweh, A., A. Alzghoul, and W. Kaialy, Data mining of solubility parameters for computational prediction of drugexcipient miscibility. Drug development and industrial pharmacy, 2014. 40 (7): p. 904-909.

[12] Bottino, A., et al., The formation of microporous polyvinylidene difluoride membranes by phase separation. Journal of Membrane Science, 1991.57 (1): p. 1-20.

[13] Salman, R. K., Gelation and melting point depression in PVDF-based gel electrolytes. 2014: University of Leeds.

[14] Sanchez, I. C., Statistical thermodynamics of polymer blends. Vol. 1. 1999: Wiley: New York.

[15] Redelius, P., Bitumen Solubility Model Using Hansen Solubility Parameter. Energy \& Fuels, 2004. 18 (4): p. 1087 1092.

[16] Belmares, M., et al., Hildebrand and Hansen solubility parameters from Molecular Dynamics with applications to electronic nose polymer sensors. Journal of Computational Chemistry, 2004. 25 (15): p. 1814-1826.

[17] Gharagheizi, F., New procedure to calculate the Hansen solubility parameters of polymers. Journal of Applied Polymer Science, 2007. 103 (1): p. 31-36.
[18] Song, J. Y., Y. Y. Wang, and C. C. Wan, Review of gel-type polymer electrolytes for lithium-ion batteries. Journal of Power Sources, 1999. 77 (2): p. 183-197.

[19] Stephan, A. M., Review on gel polymer electrolytes for lithium batteries. European Polymer Journal, 2006. 42 (1): p. 21-42.

[20] Bottino, A., et al., Solubility parameters of poly (vinylidene fluoride). Journal of Polymer Science Part B: Polymer Physics, 1988. 26 (4): p. 785-794.

[21] Grause, G., et al., Solubility parameters for determining optimal solvents for separating PVC from PVC-coated PET fibers. Journal of Material Cycles and Waste Management, 2015: p. 1-11.

[22] Merk, W., R. N. Lichtenthaler, and J. M. Prausnitz, Solubilities of fifteen solvents in copolymers of poly (vinyl acetate) and poly (vinyl chloride) from gas-liquid chromatography. Estimation of polymer solubility parameters. The Journal of Physical Chemistry, 1980. 84 (13): p. 1694-1698.

[23] <Membranes-Processing-Guide_EN-228113.PDF>.

[24] Zhang, Q. M., Bharti, V., Kavarnos, G., Schwartz, M., Poly (Vinylidene Fluoride) (PVDF) and its Copolymers. Encyclopedia of Smart Materials, 2002. 1-2: p. 807-825.

[25] Heiji, K., The Piezoelectricity of Poly (vinylidene Fluoride). Japanese Journal of Applied Physics, 1969. 8 (7): p. 975.

[26] Nunes, S. P. and K. V. Peinemann, Membrane Technology: in the Chemical Industry. 2006: Wiley.

[27] Fong, R., U. von Sacken, and J. R. Dahn, Studies of Lithium Intercalation into Carbons Using Nonaqueous Electrochemical Cells. Journal of the Electrochemical Society, 1990. 137 (7): p. 2009-2013.

[28] Moumouzias, G., et al., A study in conductances and physical constants of LiBF4 plus propylene carbonate-diethyl carbonate system. Journal of Power Sources, 1999. 81: p. 830832.

[29] Hayamizu, K., Temperature Dependence of Self-Diffusion Coefficients of Ions and Solvents in Ethylene Carbonate, Propylene Carbonate, and Diethyl Carbonate Single Solutions and Ethylene Carbonate + Diethyl Carbonate Binary Solutions of LiPF6 Studied by NMR. Journal of Chemical \& Engineering Data, 2012.57 (7): p. 2012-2017.

[30] Evchuk, I. Y., et al., Solubility of polymethyl methacrylate in organic solvents. Russian journal of applied chemistry, 2005. 78 (10): p. 1576-1580.

[31] Asmussen, F. A. U., K., Koll. Z, 1968. 223 (no. 1): p. pp. 613.

[32] Wypych, G., Handbook of Solvents. 2001: ChemTec.

[33] Iovleva, M. M., V. N. Smirnova, and G. A. Budnitskii, The Solubility of Polyacrylonitrile. Fibre Chemistry, 2001. 33 (4): p. 262-264.

[34] Bashir, Z., S. P. Church, and D. M. Price, The formation of polymer-solvent complexes of polyacrylonitrile from organic solvents containing carbonyl groups. Acta Polymerica, 1993. 44 (5): p. 211-218. 